\title{
Molecular and phenotypic characterization of clinical isolates belonging to a KPC-2- producing strain of ST15 Klebsiella pneumoniae from a Vietnamese pediatric hospital
}

Björn Berglund ${ }^{1}$, Ngoc Thi Bich Hoang ${ }^{2}$, Maria Tärnberg ${ }^{1}$, Ngai Kien Le ${ }^{2}$, Maud Nilsson', Dung Thi Khanh Khu ${ }^{2,3}$, Olov Svartström ${ }^{4}$, Jenny Welander ${ }^{4}$, Lennart E. Nilsson ${ }^{1}$, Linus Olson ${ }^{3,5^{*}}$ (D) Tran Minh Dien ${ }^{2}$, Hai Thanh Le ${ }^{2,3}$, Mattias Larsson ${ }^{3,5}$ and Håkan Hanberger ${ }^{1,3}$

\begin{abstract}
Background: Carbapenem-resistant Klebsiella pneumoniae are becoming increasingly common in hospital settings worldwide and are a source of increased morbidity, mortality and health care costs. The global epidemiology of carbapenem-resistant $K$. pneumoniae is characterized by different strains distributed geographically, with the strain ST258 being predominant in Europe and USA, and ST11 being most common in East Asia. ST15 is a less frequently occurring strain but has nevertheless been reported worldwide as a source of hospital outbreaks of carbapenemresistant K. pneumoniae.
\end{abstract}

Methods: In this study, whole-genome sequencing and antimicrobial susceptibility testing was used to characterize 57 clinical isolates of carbapenem-resistant $K$. pneumoniae belonging to a strain of ST15, which were collected at a Vietnamese pediatric hospital from February throughout September 2015.

Results: Aside from the carbapenem resistance gene b/a $a_{\mathrm{KPC}-2}$, which was carried by all isolates, prevalence of resistance genes to other antibiotics including aminoglycosides, macrolides, quinolones, fosfomycin and trimethoprim, was also high. All isolates were multidrug-resistant. Susceptibility was highest to ceftazidime/avibactam (96\%), gentamicin (91\%) and tigecycline (82\%). Notably, the colistin resistance rate was very high (42\%). Single-nucleotide polymorphism analysis indicated that most isolates belonged to a single clone.

Conclusions: The diverse variety of antibiotic resistance genes and the high antibiotic resistance rates to last-resort antibiotics such as carbapenems and colistin, is indicative of a highly adaptable strain. This emphasizes the importance of implementation of infection controls measures, continued monitoring of antibiotic resistance and prudent use of antibiotics to prevent further selection of resistant strains and the emergence of pan-resistant clones.

Keywords: Antibiotic resistance, Carbapenems, Klebsiella pneumoniae, Vietnam, Whole-genome sequencing

\footnotetext{
* Correspondence: linus.olson@ki.se

${ }^{3}$ Training and Research Academic Collaboration (TRAC)- Sweden - Vietnam, Hanoi, Vietnam

${ }^{5}$ Karolinska Institutet, Stockholm, Sweden

Full list of author information is available at the end of the article
}

(c) The Author(s). 2019 Open Access This article is distributed under the terms of the Creative Commons Attribution 4.0 International License (http://creativecommons.org/licenses/by/4.0/), which permits unrestricted use, distribution, and reproduction in any medium, provided you give appropriate credit to the original author(s) and the source, provide a link to the Creative Commons license, and indicate if changes were made. The Creative Commons Public Domain Dedication waiver (http://creativecommons.org/publicdomain/zero/1.0/) applies to the data made available in this article, unless otherwise stated. 


\section{Introduction}

Emerging multidrug-resistant gram-negative bacteria are a global problem causing increased morbidity, mortality and health care costs, which threaten the modern medical system. Particularly problematic is the dissemination of genes encoding carbapenem resistance among Klebsiella pneumoniae, with increasing prevalence reported in Europe [1], USA [2] and China [3]. The global epidemiology of carbapenem-resistant $K$. pneumoniae (CR-KP) is characterized by the spread of strains with mainly KPC-, NDM- and OXA-48-carbapenemases, and the distribution of strains differ geographically. The ST258 clonal group is dominating in Europe and USA whereas ST11 is more common in East Asian countries. ST15 is another $K$. pneumoniae clonal group associated with production of extended-spectrum $\beta$-lactamases (ESBLs) and carbapenemases and has been indicated in clinical cases and hospital outbreaks worldwide [4]. Examples include isolates with KPC-2 in Bulgaria [5], KPC-3producers in Portugal [6], OXA-48-producing isolates in Spain [7] and Vietnam [8], isolates with OXA-232 in China [9] and NDM-1-producing isolates in Nepal [10]. While ST15 isolates in specific outbreaks can be highly homogenic $[9,11]$, a high variability in antibiotic susceptibility and antibiotic resistance determinants among ST15 isolates from different study locations are indicative of a group of bacteria with high potential for horizontal gene acquisition.

In 2012 and 2013 we conducted monthly point prevalence surveys at intensive care units (ICUs) in 16 Vietnamese hospitals. The results in the adult ICUs, with 3287 cases, showed a hospital-acquired infection prevalence of $29.5 \%$, most frequently caused by Acinetobacter baumannii, Pseudomonas aeruginosa, and K. pneumoniae, with carbapenem resistance rates of 89.2, 55.7, and $14.9 \%$ respectively [12]. In the ICUs at three pediatric referral hospitals, 1363 cases were surveyed. Hospitalacquired infections were diagnosed in one third of the cases, most commonly caused by $K$. pneumoniae, among which the carbapenem resistance rate was 55\% [13]. This initiated further investigation of the epidemiology of CR-KP isolated from patients at one of the abovementioned hospitals during 2015 using whole-genome sequencing (WGS). Screening of all isolates collected during 2015 for carbapenem- and colistin-resistant isolates of $K$. pneumoniae revealed that all isolates $(n=31)$ with this antibiotic resistance phenotype belonged to a genetically homogenous strain of ST15 showing a high heterogeneity in mutations engendering colistin resistance [14]. The possibility of a hospital outbreak caused by this ST15 strain prompted further screening for ST15 among carbapenem-resistant but colistin-susceptible isolates. The aim with this study was to elucidate the epidemiology, antimicrobial susceptibility and molecular characteristics of a ST15 strain of CR-
KP possibly responsible for a clonal outbreak at a large Vietnamese pediatric hospital.

\section{Materials and methods Clinical isolates and patient data}

This study was carried out at a large pediatric hospital in Vietnam. From February throughout September 2015, all clinical isolates of $K$. pneumoniae obtained from diagnostic routine at the hospital were tested for resistance to imipenem and meropenem with antimicrobial susceptibility testing by using the VITEK 2 system (BioMérieux, Marcy-l'Étoile, France). WGS was carried out among imipenem- and meropenem-resistant isolates to elucidate the dissemination of a CR-KP ST15 strain among the patients at the hospital. In addition to 22 isolates of colistinresistant CR-KP ST15 collected from 22 patients during this period for a previous study [14], 35 unique isolates of ST15 CR-KP were further determined in the current study. A total of 57 unique isolates from 57 unique patients were thus included for further study. Partial background information collected included dates of admission and discharge, date of birth, whether the patient was originally hospitalized at the study hospital or transferred from another hospital, diagnosis, outcome, date of culture, intubation, sex, specimen, and department at which the patient was interred.

\section{DNA extraction, library preparation and whole-genome sequencing}

DNA was extracted using the EZ1 DNA Tissue Kit and the EZ1 Advanced XL instrument (Qiagen, Hilden, Germany). The extracted DNA was stored in $-20^{\circ} \mathrm{C}$ before subsequent use in library preparation. A sequencing library was constructed using Nextera XT kit (Illumina, San Diego, CA). As input DNA, $1 \mathrm{ng}$ of genomic DNA was used. The DNA concentration, determined by a Qubit 2.0 Fluorometer (Thermo Fisher Scientific, Waltham, MA), was adjusted to $0.2 \mathrm{ng} / \mu \mathrm{l}$ in RNAse-free water. Sequencing was performed on a MiSeq instrument (Illumina), and produced a mean sequencing depth of 32x.

\section{Genome assembly and bioinformatic analysis}

Genome assembly and subsequent bioinformatic analyses were performed with CLC Genomics Workbench v.9.5.3 (Qiagen). Raw reads were uploaded to the Sequence Read Archive at NCBI (Accession numbers: SRR6656601SRR6656635). Determination of antibiotic resistance genes and multilocus sequence typing was performed by querying the databases at the Center for Genomic Epidemiology (http://www.genomicepidemiology.org/). The virulence gene querying and capsule typing via the $w z i$ gene was performed using the Institut Pasteur MLST and whole genome MLST databases (http://bigsdb.pasteur.fr/). Variants were called against a reference genome (NC_022566) 
if they had a sequencing depth of $\geq 10 x$, a frequency of $\geq 90 \%$ and a Phred score $\geq 20$, and the resulting variants were used to determine the genetic relationship between the isolates using the single nucleotide polymorphism (SNP) analysis tool in CLC Genomics Workbench. Criteria for including SNPs in the phylogenetic tree were set to a sequencing depth of $\geq 10 x$, a distance between SNPs of $\geq 10 \mathrm{bp}$ and a $Z$-score $\geq 1.96$. Due to low average sequencing depth $(<15 \mathrm{x})$, samples VN26 and VN918 were excluded from the analysis.

\section{Antimicrobial susceptibility testing}

Carbapenem resistance for the CR-KP ST15 isolates were confirmed by determining MICs of meropenem by using Etests (BioMérieux) on Muller-Hinton agar (Oxoid, Basingstoke, England). Furthermore, Etests were used to determine MICs for the antibiotics ertapenem, imipenem, amikacin, gentamicin, tobramycin, fosfomycin, chloramphenicol, trimethoprim/sulfamethoxazole, ciprofloxacin and tigecycline. MICs of colistin were determined by using the broth microdilution method with cation-adjusted BBL Mueller-Hinton broth (BD, Franklin Lakes, NJ, USA) and Sensititre plates (Thermofisher Scientific, Waltham, MA, USA). Antimicrobial susceptibility was determined using the clinical breakpoints from the European Committee for Antimicrobial Susceptibility Testing (EUCAST, 2017). Isolates were considered multidrug-resistant if non-susceptible to antibiotics in three or more groups of antibiotics [15].

\section{Results}

\section{Patient data}

Partial patient data were collected from a total of 57 unique patients from which the 57 analyzed isolates originated. 28\% $(n=16)$ of the patients were female. The median age based on data available from 56 (98\%) patients was 36.0 days. The average duration of hospital stay based on available data from 49 (86\%) patients was 44.6 days. The baseline data on the patients from which the samples were collected are presented in Table 1. Data on patient outcome was available for $29(51 \%)$ of the patients included in the study. The mortality among these patients was $14 \%$. Among the 27 patients with missing outcome data, 19 were withdrawn from treatment due to pessimal prognosis with no further treatment option. Data on patient origin (community or transfer from other hospital) was available for 41 (72\%) patients, $22 \%$ of whom originated from the community whereas $78 \%$ had been transferred from other hospitals. Data on patient diagnoses were available for 53 (93\%) patients. The most common diagnoses among these patients were respiratory failure (32\%), pneumonia (28\%) and cardiovascular disease (15\%). A total of four patients (7\%) were diagnosed with septicemia, one of which had an isolate of $K$. pneumoniae isolated from their blood. The samples from which the
Table 1 Baseline parameters for the 57 patients at a large Vietnamese pediatric hospital included in the study, in relation to treatment outcome, from which carbapenem-resistant ST15 K. pneumoniae were isolated

\begin{tabular}{|c|c|c|c|c|}
\hline & Total & Discharged & Death & N.A. ${ }^{a}$ \\
\hline \multicolumn{5}{|l|}{ Sex } \\
\hline Female & 16 & 9 & 0 & 7 \\
\hline Male & 41 & 16 & 4 & 21 \\
\hline \multicolumn{5}{|l|}{ Diagnosis } \\
\hline Respiratory failure & 17 & 7 & 2 & 8 \\
\hline Pneumonia & 15 & 8 & 1 & 6 \\
\hline Cardiovascular disease & 8 & 4 & 2 & 2 \\
\hline Septicemia & 5 & 2 & 2 & 1 \\
\hline Pre-term & 5 & 2 & 2 & 1 \\
\hline Enterocolitis/peritonitis & 3 & 2 & 0 & 1 \\
\hline Esophagus atresia & 3 & 1 & 0 & 2 \\
\hline Cerebral hemorrhage & 2 & 1 & 0 & 1 \\
\hline Diaphragmatic hernia & 2 & 0 & 0 & 2 \\
\hline Other & 7 & 3 & 0 & 4 \\
\hline N.A. & 4 & 0 & 0 & 4 \\
\hline \multicolumn{5}{|l|}{ Origin } \\
\hline Other hospital & 32 & 17 & 1 & 14 \\
\hline Community & 9 & 3 & 2 & 4 \\
\hline N.A. & 16 & 5 & 1 & 10 \\
\hline \multicolumn{5}{|l|}{ Department } \\
\hline General ICU & 8 & 3 & 0 & 5 \\
\hline Neonatal ICU & 28 & 16 & 1 & 11 \\
\hline Surgical ICU & 17 & 5 & 3 & 9 \\
\hline Other & 4 & 1 & 0 & 3 \\
\hline \multicolumn{5}{|l|}{ Invasive procedure } \\
\hline Intubation & 45 & 18 & 4 & 23 \\
\hline Central vascular catheter & 36 & 13 & 4 & 19 \\
\hline Urinary catheter & 20 & 6 & 2 & 12 \\
\hline N.A. & 4 & 0 & 0 & 4 \\
\hline \multicolumn{5}{|l|}{ Specimen } \\
\hline Tracheal fluid & 35 & 13 & 3 & 19 \\
\hline Nasopharynx & 12 & 7 & 0 & 5 \\
\hline Blood & 3 & 1 & 0 & 2 \\
\hline Other & 6 & 4 & 1 & 1 \\
\hline
\end{tabular}

ICU intensive care unit

${ }^{\text {a }}$ Data not available

isolates were collected were tracheal fluid (61\%), nasopharynx (23\%), blood (5\%) and other specimens (11\%).

\section{Antibiotic resistance genes, virulence genes and wzi- typing}

Data on antibiotic resistance genes are presented in Table 2. All isolates carried the carbapenemase gene 
$b l a_{\mathrm{KPC}-2}$, however, two isolates also carried $b l a_{\mathrm{NDM}-1}$. Carriage rates of beta-lactamase gene bla $a_{\text {OXA-9, }}$, aminoglycoside resistance genes $a a c$ (6')-Ib and $a a d A 1$, fosfomycin resistance gene fos $A$, trimethoprim resistance gene $d f r A 23$ and quinolone resistance genes $o q x A$ and $o q x B$ were also high (>90\%). The ribosomal methylase gene $r m t B$, which confer high-grade resistance to aminoglycosides, was detected in five isolates (9\%). All isolates carried the mrk operon mrkABCDFJ coding for type 3 fimbriae, with four isolates additionally carrying the chromosomal $m r k$ operon transcriptional activator genes $m r k I$ and $m r k H$. The aerobactin siderophore-encoding operon iucABCD-iutA was detected in 48 out of the 57 isolates (84\%). All isolates carried the iron uptake system encoded by the $k f u A, k f u B$ and $k f u C$ genes. $r m p A 2$, a gene encoding a transcriptional activator for capsular polysaccharide synthesis, was detected in one isolate, VN956B. Because of an insertion causing a frameshift, the predicted gene product of $r m p A 2$ in $\mathrm{VN} 956 \mathrm{~B}$ was only 73 aa long as compared to the 212 aa long wild type protein. This mutation likely resulted in a dysfunctional gene product. The capsular serotype as determined through wzi-typing, was determined to be $\mathrm{K} 10$ for all isolates except isolates VN915 and VN918, for which no serotype could be determined.

\section{Antimicrobial susceptibility testing}

Data on antimicrobial susceptibility, $\mathrm{MIC}_{50}$ and $\mathrm{MIC}_{90}$ values are presented in Table 3. All isolates were multidrug-resistant. Susceptibility was highest to the antibiotics ceftazidime/avibactam (96\%), gentamicin (91\%), tigecycline (82\%), trimethoprim/sulfamethoxazole (70\%) and fosfomycin (70\%). Susceptibility was low to colistin
(58\%) and chloramphenicol (3.5\%), and $0 \%$ for the other tested antibiotics.

\section{Single-nucleotide polymorphism analysis}

The genetic relatedness of the isolates was determined with a SNP-analysis (Fig. 1). Due to low average sequencing depth $(<15 \mathrm{x})$, samples VN26 and VN918 were excluded from the analysis. The remaining 55 isolates formed two clusters consisting of 50 and 5 isolates respectively, with a minimum divergence between the clusters of 28 SNPs. The maximum divergence between the isolates in the larger clusters was 12 SNPs whereas the maximum divergence in the smaller cluster was 3 SNPs.

\section{Discussion}

Characterization by using WGS data and antimicrobial susceptibility testing was carried out on 57 clinical isolates of CR-KP of ST15 collected at a Vietnamese pediatric hospital during 8 months in 2015 in order to characterize the strain in terms of antibiotic resistance, virulence and genetic relatedness. A shortcoming of the study is that the patient data were retrospectively collected and tracing the spread of ST15 via patients and staff was impossible to perform due to the unavailability of data.

The antibiotic resistance gene carriage varied between the isolates, likely due to carriage of different antibiotic resistance plasmids, although a few antibiotic resistance genes were shared amongst the majority of isolates, for example, $b l a_{\mathrm{KPC}-2}$, $a a c\left(6^{\prime}\right)$-Ib and dfrA23. bla $a_{\mathrm{KPC}-2}$ was the dominant carbapenemase gene and was carried by all isolates. Two isolates also carried $b l a_{\mathrm{NDM}-1}$ in addition to $b l a_{\mathrm{KPC}-2}$. Heterogeneity among antibiotic resistance

Table 2 Genotypic features of 57 carbapenem-resistant ST15 K. pneumoniae isolated at a large Vietnamese hospital. Percentages are denoted in parenthesis

\begin{tabular}{|c|c|c|c|c|c|}
\hline Genes & Rate & Function & Genes & Rate & Function \\
\hline$b / a_{\mathrm{KPC}-2}$ & $57(100)$ & $\beta$-lactam resistance & $m p h(A)$ & $39(68)$ & Macrolide resistance \\
\hline$b / a_{N D M-1}$ & $2(4)$ & $\beta$-lactam resistance & $a a c\left(6^{\prime}\right)-1 \mathrm{~b}$ & $52(91)$ & Aminoglycoside resistance \\
\hline$b / a_{\mathrm{SHV}-12}$ & $36(63)$ & $\beta$-lactam resistance & aadAl & $52(91)$ & Aminoglycoside resistance \\
\hline$b / a_{\mathrm{SHV}-28}$ & $48(84)$ & $\beta$-lactam resistance & $\operatorname{aph}\left(3^{\prime \prime}\right)-1 \mathrm{~b}$ & $8(14)$ & Aminoglycoside resistance \\
\hline bla $a_{\mathrm{SHV}-73}$ & $6(11)$ & $\beta$-lactam resistance & $a p h(6)-I d$ & $8(14)$ & Aminoglycoside resistance \\
\hline$b / a_{\mathrm{SHV}-155}$ & $1(2)$ & $\beta$-lactam resistance & $r m t B$ & $5(9)$ & Aminoglycoside resistance \\
\hline$b / a_{\text {TEM-1B }}$ & $16(28)$ & $\beta$-lactam resistance & sul1 & $8(14)$ & Sulphonamide resistance \\
\hline$b / a_{\text {TEM-199 }}$ & $36(63)$ & $\beta$-lactam resistance & sul2 & $8(14)$ & Sulphonamide resistance \\
\hline$b l a_{\text {OXA-9 }}$ & $52(91)$ & $\beta$-lactam resistance & dfrA16 & $8(14)$ & Trimethoprim resistance \\
\hline fos $A$ & $57(100)$ & Fosfomycin resistance & dfrA23 & $56(98)$ & Trimethoprim resistance \\
\hline fOSA3 & $5(9)$ & Fosfomycin resistance & iucABCD-iutA & $48(84)$ & Iron-acquisition \\
\hline oqxA & $57(100)$ & Quinolone resistance & mrk operon & $57(100)$ & Adhesion \\
\hline$o q \times B$ & $57(100)$ & Quinolone resistance & $k f u A B C$ & $57(100)$ & Iron-uptake \\
\hline $\mathrm{Cm} / \mathrm{A} 1$ & $8(14)$ & Phenicol resistance & $r m p A 2$ & $1(2)$ & Regulator of mucoid phenotype \\
\hline
\end{tabular}


Table 3 Antimicrobial susceptibility pattern of 57 isolates of carbapenem-resistant ST15 K. pneumoniae isolated at a large Vietnamese pediatric hospital

\begin{tabular}{|c|c|c|c|c|c|c|}
\hline Antimicrobial agent & $\mathrm{S} \leq(\mathrm{mg} / \mathrm{L})$ & $\mathrm{R}>(\mathrm{mg} / \mathrm{L})$ & $\mathrm{S}(\%)$ & $\mathrm{R}(\%)$ & $\mathrm{MIC}_{50}(\mathrm{mg} / \mathrm{L})$ & $\mathrm{MIC}_{90}(\mathrm{mg} / \mathrm{L})$ \\
\hline Gentamicin & 2 & 4 & 91 & 8.8 & 1.5 & 2 \\
\hline Tobramycin & 2 & 4 & 0 & 100 & 16 & 24 \\
\hline Amikacin & 8 & 16 & 0 & 96 & 32 & 64 \\
\hline Chloramphenicol & 8 & 8 & 3.5 & 96 & 128 & $>256$ \\
\hline Trimethoprim/sulfamethoxazole & 2 & 4 & 70 & 25 & 2 & $>32$ \\
\hline Ciprofloxacin & 0.25 & 0.5 & 0 & 100 & $>32$ & $>32$ \\
\hline Cefotaxime & 1 & 2 & 0 & 100 & $>32$ & $>32$ \\
\hline Imipenem & 2 & 8 & 0 & 96 & $>32$ & $>32$ \\
\hline Meropenem & 2 & 8 & 0 & 100 & $>32$ & $>32$ \\
\hline Ertapenem & 0.5 & 1 & 0 & 100 & $>32$ & $>32$ \\
\hline Tigecycline & 1 & 2 & 82 & 7.0 & 0.5 & 2 \\
\hline Fosfomycin & 32 & 32 & 70 & 30 & 16 & $>1024$ \\
\hline Ceftazidime/avibactam & 8 & 8 & 96 & 3.5 & 2 & 3 \\
\hline Colistin & 2 & 2 & 58 & 42 & 0.5 & 32 \\
\hline
\end{tabular}

genes and carbapenemase genes in particular are reflected in previous publications on ST15 outbreaks worldwide. Examples of reports include a clinical outbreak of ST15 K. pneumoniae with KPC-2 in Bulgaria [5], and NDM-1producing ST15 in a Nepalese hospital outbreak [10]. In another ST15 outbreak at a hospital in Vietnam, the isolates carried predominantly bla $a_{\text {OXA-48 }}$ (89\%) but a few had $b l a_{\mathrm{NDM}-4}(11 \%)$ [8]. The varied array of antibiotic resistance genes observed among the individual ST15 isolates in this study as well as in comparison with other studies on ST15 K. pneumoniae [5, 8, 10, 11] suggests a strain with a high capacity for horizontal gene transfer and, in particular, a propensity for acquisition of antibiotic resistance genes.

Susceptibility rates were highest for the antibiotics ceftazidime/avibactam (96\%) and gentamicin (91\%).

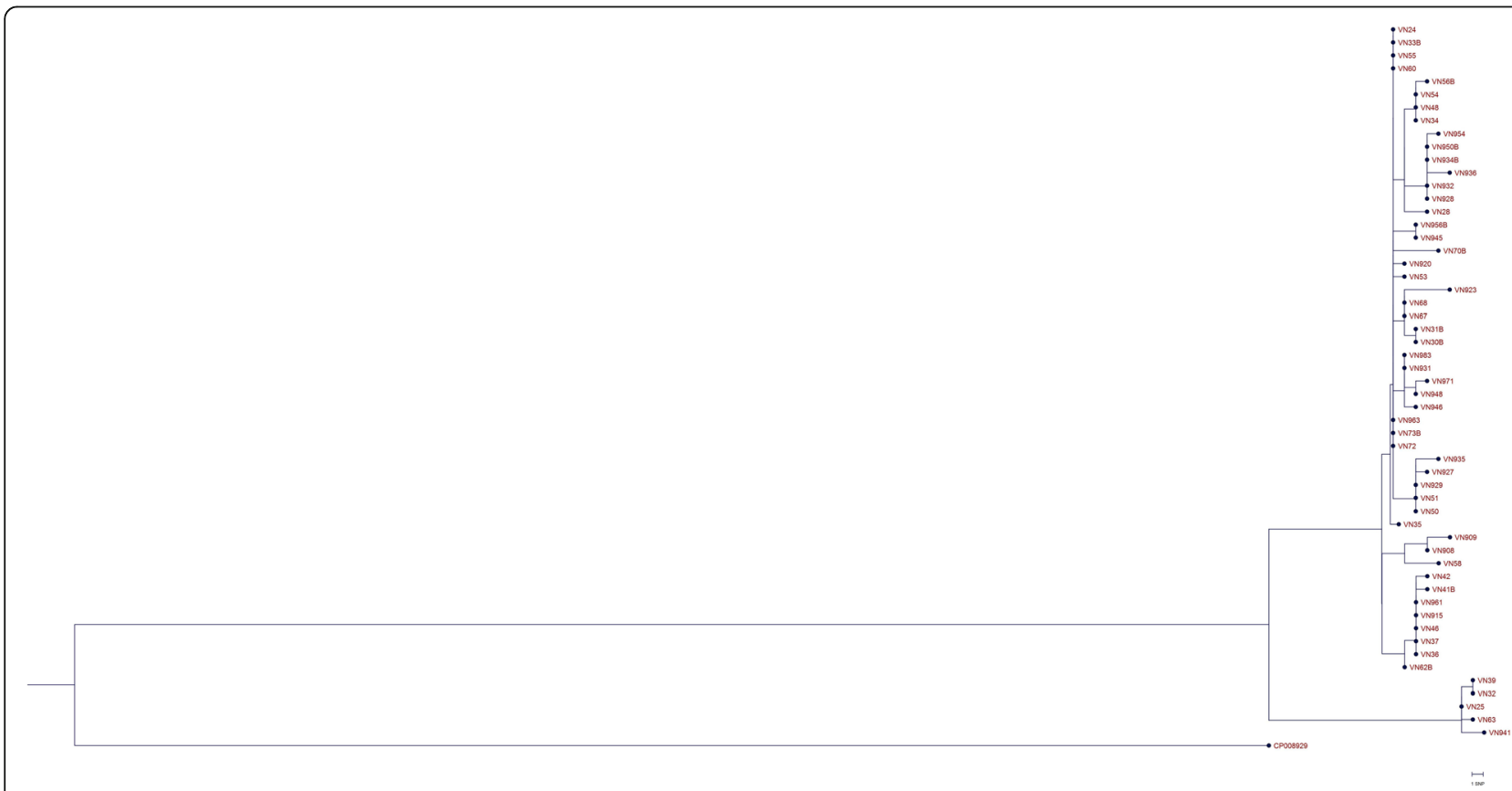

Fig. 1 Single-nucleotide polymorphism analysis of 55 isolates of carbapenem-resistant $K$. pneumoniae isolated at a large Vietnamese hospital. Due to low average sequencing depth $(<15 \mathrm{x})$, samples VN26 and VN918 were excluded from the analysis. An ST15 K. pneumoniae (NCBI accession no: CP008929) isolated from blood at a Nepali hospital [16] was used as an outlier 
Ceftazidime/avibactam is not approved for use in Vietnam and there are yet no international data supporting neonatology use. This indicates the urgent need, in the current epidemiological situation, for clinical trials evaluating treatment with antibiotic combinations as well as ceftazidime/ avibactam in the patient group. However, two isolates carrying the carbapenemase gene $b l a_{\mathrm{NDM}-1}$ showed high levels of resistance to ceftazidime/avibactam (MIC $>256 \mathrm{mg} / \mathrm{L}$ ). Although none of the isolates in the study were susceptible to the aminoglycosides amikacin and tobramycin, likely due to the high rates of the aminoglycoside resistance genes aac (6')-Ib and aadA1, all but five isolates were susceptible to gentamicin. These five gentamicin-resistant isolates carried the ribosomal methylase-gene $r m t B$ and showed high levels of resistance to all tested aminoglycosides (MIC $>256 \mathrm{mg} / \mathrm{L}$ ). While ceftazidime/avibactam and gentamicin showed the highest efficacy against the isolates in this study, overuse of either of these antibiotics may select for these corresponding resistance genes (i.e., $b l a_{\mathrm{NDM}-1}$ and $r m t B)$ and facilitate dissemination of the carrier genetic elements, further exacerbating the antibiotic resistance situation.

The rates of susceptibility to ciprofloxacin and chloramphenicol were very low, 0 and $3.5 \%$ respectively. Although chloramphenicol is not commonly used in Vietnam any longer, particularly among the type of patients included in the current study, fluoroquinolones are commonly used at Vietnamese ICUs [12]. The high resistance rate to ciprofloxacin (100\%) emphasize the need for antimicrobial susceptibility testing to inform effective treatment. Trimethoprim/sulfamethoxazole and fosfomycin susceptibilities were $70 \%$ to both antibiotics. For trimethoprim/sulfamethoxazole, 17 isolates were resistant. High levels of resistance (MIC $>32 \mathrm{mg} / \mathrm{L}$ ) was displayed by eight isolates, all of which carried sul1 and sul2 (sulphonamide resistance genes), and $d f r A 16$ and dfrA23 (trimethoprim resistance genes).

The colistin resistance rate among the studied ST15 isolates was high (42\%) and is a cause of major concern since colistin combination treatment is the most effective treatment option against CR-KP infections available today in Vietnam. The colistin resistance mechanisms of the 21 colistin-resistant isolates have been reported on elsewhere [14]. The resistance phenotype in these isolates could be attributed to chromosomal changes in $m g r B$, a known loss-of-function colistin resistance gene. Among these isolates, seven different genetic events in $m g r B$ predicted to lead to disrupted functionality of the gene product were observed. The high genetic relatedness of the colistin-susceptible and colistin-resistant ST15 isolates combined with the high heterogeneity in mutations leading to resistance in the resistant isolates, are indicative of a strain exposed to selection pressure via colistin, possibly in the hospital environment. None of the plasmid-mediated colistin resistance genes of the mor family could be detected among the ST15 isolates in this study, nevertheless, $m c r-1$ has previously been reported in three isolates of CR-KP of ST307 at the study hospital [17]. Continued resistance-monitoring and prudent use of colistin are imperative to prevent dissemination of $m c r-1$ and further emergence and selection of colistin resistance-engendering mutations in this hospital setting.

All isolates were determined to carry the mrkABCDFJ operon encoding the type 3 fimbriae system, a virulence factor that is associated with biofilm formation and enhanced capacity for binding to abiotic surfaces, which could enhance the survival of the strain in hospital environments and increase its capacity for invasiveness via for example endotracheal tubes and catheters. $84 \%$ of the isolates carried the operon encoding the aerobactin siderophore. Production of aerobactin is overrepresented among hypervirulent strains of K. pneumoniae, however, the siderophore does not appear to be a factor by itself sufficient to engender the hypervirulence phenotype and the operon is also present among non-hypervirulent strains albeit at lower ratios [18].. One isolate carried $\operatorname{rmp} A 2$, a gene associated to hypervirulence. However, mutations in this gene resulted in a heavily truncated, and likely dysfunctional protein. The capsular serotype was determined to be $\mathrm{K} 10$ for 55 isolates, a serotype not typically associated to hypervirulence. Serotypes of the remaining two isolates (VN915B and VN918) were indeterminable, likely due to assembly or sequencing problems. Given the data presented above, it is unlikely that any of the isolates analyzed in this study could be considered hypervirulent. Most of the isolates were from tracheal fluid or nasopharynx (84\%), which could indicate colonization rather than infection. The mortality rate was $14 \%$, however, 19 patients were withdrawn from medical treatment due to pessimal prognosis. Although follow-up information for these patients were not available, the outcome for withdrawn patients is usually death, meaning the actual mortality rate likely lie in the range of $14-48 \%$. The patient group studied had high rates of co-morbidities, and colonization with CR-KP may not have been a factor in the patient outcome in several cases. Nevertheless, colonization with carbapenem-resistant Enterobacteriales has been shown to increase the risk of subsequent infection with the pathogen [19], and the isolates in the current study were collected from ICUs and neonatal wards, where the patients are likely to be immunologically vulnerable. This may enhance the capacity of this strain to be invasive, as also indicated by the fact that four isolates were recovered from blood.

The SNP-analysis showed that the isolates were closely related genetically, but clustered into two sub clusters (Fig. 1) constituted of 50 and 5 isolates respectively separated by 28 SNPs. The low maximum divergence within 
each cluster were, 12 and 3 SNPs respectively, indicate that these clusters constitute two clones [20]. The five isolates constituting the smaller cluster were homogenous in terms of antibiotic susceptibilities, the exception being VN941 which was resistant to colistin (MIC $=16 \mathrm{mg} / \mathrm{L}$ ). The colistin resistance in this isolate was due to an insertion sequence transposition into the $\mathrm{PhoP} / \mathrm{PhoQ}$ two component system regulatory gene $m g r B$ as previously reported [14]. Antibiotic resistance gene carriage in the isolates were also similar, except for VN941 which alone in the cluster carried $a a c$ (6')-Ib and lacked bla ${ }_{\text {TEM-1B }}$. The isolates in this cluster carried the same antibiotic resistance genes and were notably the only isolates in the study which carried the aminoglycoside resistance gene $r m t B$ and were resistant to gentamicin. However, there was a high diversity in terms of antibiotic susceptibility and carriage of antibiotic resistance genes in the isolates within the clusters. This indicates that the clones might have disseminated throughout the hospital and over time taken up plasmids containing antibiotic resistance genes. Strains of ST15 have previously been shown to have a high propensity for dissemination and circulation in hospital environments and to cause hospital-acquired infections, as reported in hospital outbreaks in the Netherlands [11] and in Nepal [16]. A large proportion of the patients from which the isolates were collected $(78 \%$ of the patients for which data was available) were transferred from other hospitals, and so another possibility would be that this ST15 strain is well-established in many different hospital settings in Vietnam and the difference in resistance gene and plasmid carriage reflects the plasmid diversity in the local settings. Regardless of origin, there is an urgent need for infection controls measures and antibiotic stewardship to minimize transmission of and selection for this and other carbapenem-resistant strains.

\section{Conclusions}

In the current study, multidrug-resistant CR-KP belonging to the ST15 strain from a large Vietnamese pediatric hospital were characterized with WGS and antimicrobial susceptibility testing. The diverse variety of antibiotic resistance genes among the isolates and the high antibiotic resistance rate is indicative of a highly adaptable strain. This emphasizes the importance of implementation of infection control measures, continued monitoring of antibiotic resistance and prudent use of antibiotics to prevent further selection of resistant strains and the emergence of pan-resistant clones.

\section{Abbreviations}

aa: Amino acid; CR-KP: Carbapenem-resistant Klebsiella pneumoniae; ICU: Intensive care unit; SNP: Single-nucleotide polymorphism; WGS: Wholegenome sequencing

\section{Acknowledgements}

We would like to thank the Vietnam National Children's Hospital, the Ministry of Health in Vietnam, and the World Health Organization (WHO) for their help in facilitating this study.

\section{Authors' contributions}

$\mathrm{BB}, \mathrm{NKL}, \mathrm{DTKK}, \mathrm{LEN}, \mathrm{TMD}, \mathrm{HTL}, \mathrm{ML}$ and $\mathrm{HH}$ conceived of and designed the study. BB, NTBH, MT, NKL, MN, OS, JW and LO acquired the laboratory and clinical data. BB, MT, MN, OS, JW and LO analyzed the data. BB, MT, MN, JW, LEN, LO, ML and HH drafted and critically revised the manuscript. All coauthors approved of the final version of the manuscript.

\section{Funding}

This work was supported by Training and Research Academic Collaboration (TRAC) - Sweden - Vietnam financed by the Swedish Foundation for International Cooperation in Research and Higher Education (STINT) (SG 2015-5972), the Swedish Research Council (330-2014-6356), Region Östergötland (LIO-793191), Linköping University, and the Karolinska Institute.

\section{Availability of data and materials}

WGS data generated/analyzed in this study is available at the Sequence Read Archive at NCBI (https://www.ncbi.nlm.nih.gov/sra) with the accession numbers SRR6208298-SRR6208328 and SRR6656601-SRR6656635.

\section{Ethics approval and consent to participate}

Ethical approval for this study was granted by the Ethics Committee at the Vietnam National Children's Hospital (reference no. VNCH-RICH-16-014). Isolates analyzed in the study were from samples collected in the clinical routine, however, patients and their legal guardians were informed about the study and their right to opt-out of participating in the study.

Consent for publication

Not applicable.

\section{Competing interests}

The authors declare that the research was conducted in the absence of any commercial or financial relationships that could be construed as a potential conflict of interest.

\section{Author details}

${ }^{1}$ Department of Clinical and Experimental Medicine, Linköping University, Linköping, Sweden. Vietnam National Children's Hospital, Hanoi, Vietnam. ${ }^{3}$ Training and Research Academic Collaboration (TRAC) - Sweden - Vietnam, Hanoi, Vietnam. ${ }^{4}$ Department of Clinical Microbiology and Department of Clinical and Experimental Medicine, Linköping University, Linköping, Sweden. ${ }^{5}$ Karolinska Institutet, Stockholm, Sweden.

Received: 20 May 2019 Accepted: 24 September 2019 Published online: 16 October 2019

\section{References}

1. Albiger B, Glasner C, Struelens MJ, Grundmann H, Monnet DL. European Survey of Carbapenemase-Producing Enterobacteriaceae (EUSCAPE) working group. Carbapenemase-producing Enterobacteriaceae in Europe: assessment by national experts of 38 countries, May 2015. Euro Surveill. 2015;20(45).

2. Guh AY, Limbago BM, Kallen AJ. Epidemiology and prevention of carbapenem-resistant Enterobacteriaceae in the United States. Expert Rev Anti-Infect Ther. 2014;12:565-80.

3. Xu A, Zheng B, Xu YC, Huang ZG, Zhong NS, Zhuo C. National epidemiology of carbapenem-resistant and extensively drug-resistant gramnegative bacteria isolated from blood samples in China in 2013. Clin Microbiol Infect. 2016;22(Suppl 1):S1-8.

4. Lee $\mathrm{CR}$, Lee JH, Park KS, Kim YB, Jeong BC, Lee SH. Global dissemination of Carbapenemase-producing Klebsiella Pneumoniae: epidemiology, genetic context, treatment options, and detection methods. Front Microbiol. 2016;7:895.

5. Markovska R, Stoeva T, Schneider I, Boyanova L, Popova V, Dacheva D, et al. Clonal dissemination of multilocus sequence type ST15 KPC-2-producing Klebsiella pneumoniae in Bulgaria. APMIS. 2015;123:887-94. 
6. Vubil D, Figueiredo R, Reis T, Canha C, Boaventura L, Da Silva GJ. Outbreak of KPC-3-producing ST15 and ST348 Klebsiella pneumoniae in a Portuguese hospital. Epidemiol Infect. 2017;145:595-9.

7. Madueño A, González García J, Fernández-Romero S, Oteo J, Lecuona M. Dissemination and clinical implications of multidrug-resistant Klebsiella pneumoniae isolates producing OXA-48 in a Spanish hospital. J Hosp Infect. 2017;96:116-22

8. Tada T, Tsuchiya M, Shimada K, Nga TTT, Thu LTA, Phu TT, et al. Dissemination of Carbapenem-resistant Klebsiella pneumoniae clinical isolates with various combinations of Carbapenemases (KPC-2, NDM-1, NDM-4, and OXA-48) and 16S rRNA Methylases (RmtB and RmtC) in Vietnam. BMC Infect Dis. 2017;17:467.

9. Yin D, Dong D, Li K, Zhang L, Liang J, Yang Y, et al. Clonal dissemination of OXA-232 Carbapenemase-producing Klebsiella pneumoniae in neonates. Antimicrob Agents Chemother. 2017;61:e00385-17.

10. Chung The H, Karkey A, Pham Thanh D, Boinett CJ, Cain AK, Ellington M, et al. A high-resolution genomic analysis of multidrug-resistant hospital outbreaks of Klebsiella pneumoniae. EMBO Mol Med. 2015;7:227-39.

11. Zhou K, Lokate M, Deurenberg RH, Tepper M, Arends JP, Raangs EG, et al. Use of whole-genome sequencing to trace, control and characterize the regional expansion of extended-spectrum $\beta$-lactamase producing ST15 Klebsiella pneumoniae. Sci Rep. 2016;6:20840.

12. Phu VD, Wertheim HF, Larsson M, Nadjm B, Dinh QD, Nilsson LE, et al. Burden of hospital acquired infections and antimicrobial use in Vietnamese adult intensive care units. PLoS One. 2016;11:e0147544.

13. Le NK, Hf W, Vu PD, Khu DT, Le HT, Hoang BT, et al. High prevalence of hospital-acquired infections caused by gram-negative carbapenem resistant strains in Vietnamese pediatric ICUs: A multi-centre point prevalence survey. Medicine (Baltimore). 2016;95:e4099

14. Berglund B, Hoang NTB, Tärnberg M, Le NK, Svartström O, Khu DTK, et al. Insertion sequence transpositions and point mutations in mgrB causing colistin resistance in a clinical strain of carbapenem-resistant Klebsiella pneumoniae from Vietnam. Int J Antimicrob Agents. 2018;51:789-93.

15. Magiorakos AP, Srinivasan A, Carey RB, Carmeli Y, Falagas ME, Giske CG, et al. Multidrug-resistant, extensively drug-resistant and pandrug-resistant bacteria: an international expert proposal for interim standard definitions for acquired resistance. Clin Microbiol Infect. 2012;18:268-81.

16. Stoesser N, Giess A, Batty EM, Sheppard AE, Walker AS, Wilson DJ, et al. Genome sequencing of an extended series of NDM-producing Klebsiella pneumoniae isolates from neonatal infections in a Nepali hospital characterizes the extent of community- versus hospital-associated transmission in an endemic setting. Antimicrob Agents Chemother. 2014;58: 7347-57.

17. Berglund B, Hoang NTB, Tärnberg M, Le NK, Welander J, Nilsson M, et al. Colistin- and carbapenem-resistant Klebsiella pneumoniae carrying mcr-1 and blaOXA-48 isolated at a paediatric hospital in Vietnam. J Antimicrob Chemother. 2018;73:1100-2.

18. Paczosa MK, Mecsas J. Klebsiella pneumoniae: going on the offense with a strong defense. Microbiol Mol Biol Rev. 2016;80:629-61.

19. Giannini MA, Gilliam C, Owings A, Glover B, Gipson M, Hakim H. Does colonization with Carbapenem-resistant Enterobacteriaceae correlate to infection? Am J Infect Control. 2017:45:S37.

20. Schürch AC, Arredondo-Alonso S, Willems RLL, Goering RV. Whole genome sequencing options for bacterial strain typing and epidemiological analysis based on single nucleotide polymorphism vs gene-by-gene-based approaches. Clin Microbiol Infect. 2018;24:350-4.

\section{Publisher's Note}

Springer Nature remains neutral with regard to jurisdictional claims in published maps and institutional affiliations.

Ready to submit your research? Choose BMC and benefit from:

- fast, convenient online submission

- thorough peer review by experienced researchers in your field

- rapid publication on acceptance

- support for research data, including large and complex data types

- gold Open Access which fosters wider collaboration and increased citations

- maximum visibility for your research: over $100 \mathrm{M}$ website views per year

At BMC, research is always in progress.

Learn more biomedcentral.com/submissions 